\title{
Expression of neuro-oncological ventral antigen-1 in small-cell lung cancer and its value in pathological diagnosis
}

\author{
Shuangshuang Deng ${ }^{1}$, Meixuan Liu ${ }^{2}$, Tianyu Xiao ${ }^{1}$, Jinli Gao ${ }^{1}$ \\ ${ }^{1}$ Department of Pathology, ${ }^{2}$ Department of Respiratory Medicine, Shanghai East Hospital, Tongji University School of Medicine, Shanghai 200120, \\ China \\ Contributions: (I) Conception and design: S Deng; (II) Administrative support: J Gao; (III) Provision of study materials or patients: M Liu; (IV) \\ Collection and assembly of data: T Xiao; (V) Data analysis and interpretation: S Deng; (VI) Manuscript writing: All authors; (VII) Final approval of \\ manuscript: All authors. \\ Correspondence to: Jinli Gao. Department of Pathology, Shanghai East Hospital, Tongji University School of Medicine, \#150 Jimo Road, Shanghai \\ 200120, China. Email: gaojinli.ok@163.com.
}

Background: To investigate the expression of neuro-oncological ventral antigen-1 (Nova1) in small-cell lung cancer (SCLC) and its value in clinical pathological diagnosis of SCLCs.

Methods: Nova1 expression in the SCLC cell line H446, lung adenocarcinoma cell line A549, squamous cell carcinoma (SCC) cell line H226 and bronchial epithelial cell line BEAS-2B were examined by RT-PCR. It's expression in paraffin specimens of 100 SCLC cases, 15 specimens of lung adenocarcinoma cases and 15 specimens of lung SCC cases, as well as adjacent normal tissue was determined by immunohistochemistry. The positive rate of Nova1 in SCLC tissue was compared with those of traditional neuroendocrine markers CD56, Syn and CgA.

Results: Nova1 expression in SCLC cell line H446 was significantly higher than that of bronchial epithelial cell line BEAS-2B and lung adenocarcinoma cell line A549 and SCC cell line H226. The positive rate of Nova1 in SCLC tissue was similar to that of CD56 but higher than those of Syn and CgA. Nova1 was neither expressed in the cancer tissue nor in the adjacent normal tissue of the 15 cases of SCC and the 15 cases of adenocarcinoma. In all six cases of combined SCLC, Nova1 was expressed in the SCLC component, whereas markers of SCC or adenocarcinoma were absent.

Conclusions: Nova1 can be used as a novel neuroendocrine marker for pathological diagnosis of SCLC in clinical practice with high sensitivity and specificity, in addition to its role as an independent prognostic marker of overall survival and tumour recurrence, as suggested by previous studies.

Keywords: Nova1; small-cell lung carcinoma; immunohistochemistry

Submitted Sep 26, 2019. Accepted for publication Dec 17, 2019.

doi: $10.21037 /$ tcr.2019.12.99

View this article at: http://dx.doi.org/10.21037/tcr.2019.12.99

\section{Introduction}

Small-cell lung cancer (SCLC) was first reported by WHO in 1926 (1) as a poorly differentiated malignant tumour that is highly proliferative, invasive, metastatic and sensitive to chemotherapeutic drugs but tended to develop drug resistance (2). In 2012, 353,000 people died of lung cancer in Europe (3); SCLC accounted for 10-13\% of these deaths, and smoking was a major risk factor. SCLC originates from $\mathrm{K}$ cells of the bronchial epithelial mucous gland, which fulfil a neuroendocrine function, and its pathological types include the oat cell, intermediate and mixed types. Under the microscope, tumour cells are spindle-shaped or lymphocyte-like, with few cytoplasm and coarse particles. The cells are often densely integrated into groups, some arranged like parallel fences and shaped like oats. In clinical practice, the characteristic arrangement and cell shape may 
be destroyed due to squeezing during sampling, which makes HE sections of SCLC hardly distinguishable from lymphocyte infiltration, poorly differentiated squamous cell carcinoma (SCC), basal cell carcinoma, etc., especially when the sample is taken by fiberoptic bronchoscopy or lung biopsy, which result in a sample with fewer cancer cells. In such situations, it would be extremely important to make full use of immunohistochemical indicators for differential diagnosis. Currently, commonly used markers for SCLC include CD56, Syn and CgA. Compared to Syn and CgA, CD56 is more sensitive to SCLC, but it is also expressed in natural killer cells (NK cells), SCC, adenocarcinoma, mesothelioma, etc., so its specificity is relatively low. Most SCLCs express neuroendocrine markers; nevertheless, up to $10 \%$ of SCLC cases are negative for all three neuroendocrine markers (4). In addition, commonly used SCC markers P63, P40, etc., can be expressed in SCLCs, making its diagnosis more difficult. So, it is necessary to find a novel neuroendocrine marker with higher specificity and sensitivity for the diagnosis of SCLCs.

\section{Methods}

\section{General information}

Paraffin-embedded tissues were collected from the Department of Pathology, Shanghai Oriental Hospital from January 2016 to December 2017, all of which were biopsy or surgical resection specimens. We collected 100 samples of SCLCs, including 84 primary cases and 16 lymph node/liver metastases, and six of them were combined SCLCs. As controls, 15 samples of SCC and 15 samples of adenocarcinoma were also collected. All cases were diagnosed by two senior pathologists according to the 2015 WHO lung cancer histological classification criteria and immunohistochemistry results, and none of the cases were previously treated. All specimens were obtained with informed consent from the patient.

\section{Materials}

The major instruments required for the experiment were an RNA extraction kit (MRC, USA), a reverse transcription and qPCR kit, a cDNA synthesiser (TaKaRa, Japan), an amplification instrument (Bio-Rad, USA), a thermocycler (ABI, USA) and an ultra-micro bioassay instrument (Thermo, USA). The antibody used in the experiment was anti-Nova1 (Abcam, UK). The DAB chromogenic kit was used for immunohistochemistry (Zsbio). Human bronchial epithelial (BEAS-2B), small-cell carcinoma (H446), lung adenocarcinoma (A549) and SCC (H226) cell lines used in the experiments were purchased from the ATCC (Manassas, VA, USA).

\section{Study methods}

\section{Cell culture}

Cell lines were cultured in RPMI-1640 medium in a $37^{\circ} \mathrm{C}$ incubator with $5 \% \mathrm{CO}_{2}$.

\section{RNA extraction and quality test}

When the cell density reached approximately $80 \%$, total RNA was extracted by adding $1 \mathrm{~mL}$ of TRIzol solution. The purity and concentration of the RNA were determined by ultra-micro bioassay absorbance assay, and RNA with an $\mathrm{A}_{260} / \mathrm{A}_{280}$ between 1.8 and 2.0 was considered sufficiently pure.

\section{RT-PCR}

RT-PCR was carried out according to the kit manufacturer's ubstryctuibs, wherein reverse transcription was performed by incubation at $37^{\circ} \mathrm{C}$ for $15 \mathrm{~min}$ and $85^{\circ} \mathrm{C}$ for $5 \mathrm{~s}$. The cDNA product was used as the template for amplification. The thermocycling parameters were initial denaturation at $95{ }^{\circ} \mathrm{C}$ for $2 \mathrm{~min} ; 40$ cycles of denaturation at $94{ }^{\circ} \mathrm{C}$ for $15 \mathrm{~s}$ and extension at $60{ }^{\circ} \mathrm{C}$ for $30 \mathrm{~s}$; and a final hold at $4{ }^{\circ} \mathrm{C}$ The melting curve was drawn for quality control, and quantification was carried out by using the $2^{-\Delta \Delta C t}$ method. Three duplicate wells were set in each group, and the experiment was repeated three times. GAPDH was selected as the internal control. The corresponding PCR primers were as follows: NOVA1, forward, 5'-GGTCTCAGCCAAGCAGCAGCAA-3' and reverse, 5'-TTGCAGCAGTAGCAGCAGCCAG-3'; GAPDH, forward, 5'-TGGATCTGACATGCCGCCTGGA-3' and reverse, 5'-AGGTCCACCA CCCTGTTGCTGT-3'.

\section{Immunohistochemistry}

The above-mentioned tissue blocks were fixed with $4 \%$ formaldehyde, conventionally dehydrated before embedding in paraffin and sliced into $4-\mu \mathrm{m}$ sections Immunohistochemical of neuro-oncological ventral antigen-1 (Nova1), P63, P40, CD56, synaptophysin (Syn) and chromogranin $\mathrm{A}(\mathrm{CgA})$ were performed using the EnVision two-step method on the Dako Omnis automated 


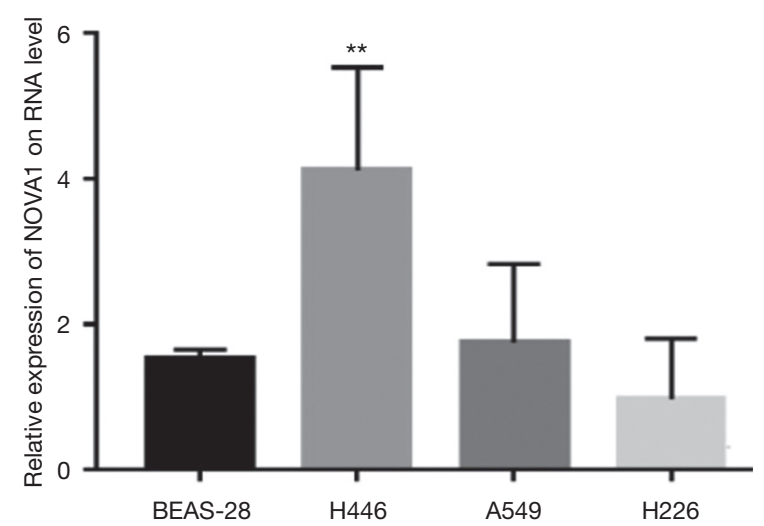

Figure 1 Nova1 mRNA was highly expressed in the SCLC H446 cell line. Note: BEAS-2B (normal bronchial epithelial cell line), H446 (SCLC cell line), A549 (lung adenocarcinoma cell line), H226 (squamous cell carcinoma cell line), ${ }^{* *}, \mathrm{P}<0.05$.

immunohistochemical stainer. All target antigens were prepared with high- pressure sodium citrate buffer at $\mathrm{pH}$ 6.0. PBS was used in place of the primary antibody for a blank control, and already known positive cases were used as positive controls. The specific steps were carried out according to the kit manufacturer's instructions. In immunohistochemical staining, Nova1 was localised in the nucleus, Syn and CgA were localised in the cytoplasm, CD56 was localised in the cell membrane and/or cytoplasm, and P63 and P40 were localised in the nucleus. For Nova1 staining, $<5 \%$ of cells stained under a $400 \times$ field was considered negative expression, $5-50 \%$ was considered low expression and $>50 \%$ was considered high expression.

\section{Statistical analysis}

Experimental data were analysed using SPSS 22.0 analysis software. Measurement data were represented as mean \pm standard deviation; comparison between two groups was performed with a Student's $t$-test. Count data were analysed by employing a Chi-square test. Results were considered statistically significant at $\mathrm{P}<0.05$.

\section{Results}

Expression of Nova1 gene at cellular level Bioinformatics analysis using the Oncomine database suggested that the expression level of Nova1 in SCLC was significantly higher than that in normal lung tissue, adenocarcinoma SCC and large-cell carcinoma. In our research, RT-PCR results demonstrated that the expression of Nova 1 mRNA in SCLC H446 cells was higher than that in normal BEAS-2B bronchial epithelial cells and lung adenocarcinoma A549 or H226 SCC cells (Figure 1).

\section{Nova1 expression in tissue samples}

\section{Expression of Nova1 in SCLC}

The positive rate of Nova1 in SCLC was $88 \%$ (Figure 2). Nova1 expression in primary and metastatic SCLC tissues was significantly higher than that in lung SCC and lung adenocarcinoma tissues. Compared to lymph node/liver metastases, Nova1 expression was significantly higher in primary SCLC $\left(\chi^{2}=6.684, \mathrm{P}=0.001\right)$, as shown in Table 1 .

\section{Expression of Nova1 in NSCLC}

Nova1 was neither expressed in the cancer tissue nor in the adjacent normal tissue of the 15 cases of SCC and the 15 cases of adenocarcinoma. Six cases of combined SCLC were included in the sample, including three cases of SCLC complicated by SCC and three cases of SCLC complicated by adenocarcinoma. Nova1 expression was detected in the SCLC components of all six cases but absent in the SCC or adenocarcinoma component, as shown in Figure 3.

\section{Positive rates of Nova1, CD56, CgA, SYN, P63 and P40 in SCLC tissues}

Positive rates of Nova1, CD56, SYN, CgA, P63 and P40 in SCLC were $88 \%$ (88/100), 92\% (92/100), 75\% (75/100), $79 \%$ (79/100), 13\% (13/100) and 7\% (7/100), respectively. The rate of Nova1 positivity was not significantly different from that of $\operatorname{CD} 56\left(\chi^{2}=0.889, P=0.336\right)$ but was significantly higher than that of Syn and $\operatorname{CgA}\left(\chi^{2}=5.604, \mathrm{P}=0.018\right.$ and $\left.\chi^{2}=2.940, \mathrm{P}=0.086\right)$. The commonly used SCC markers $\mathrm{P} 63$ and $\mathrm{P} 40$ had positivity rates of $13 \%$ and $7 \%$ in the SCLC samples, respectively. As shown in Table 2.

\section{Discussion}

\section{Expression of Nova1 in SCLC specimens}

Nova1 is a central nervous system-specific RNA-binding protein. As a self-antigen of the nervous system, it was first identified in patients with paraneoplastic opsoclonusmyoclonus ataxia (POMA) $(5,6)$. In recent years, it has been found that Nova1 plays an important role in the development of lymphoma, multiple myeloma, gastric cancer, melanoma, astrocytoma and oligodendroglioma 

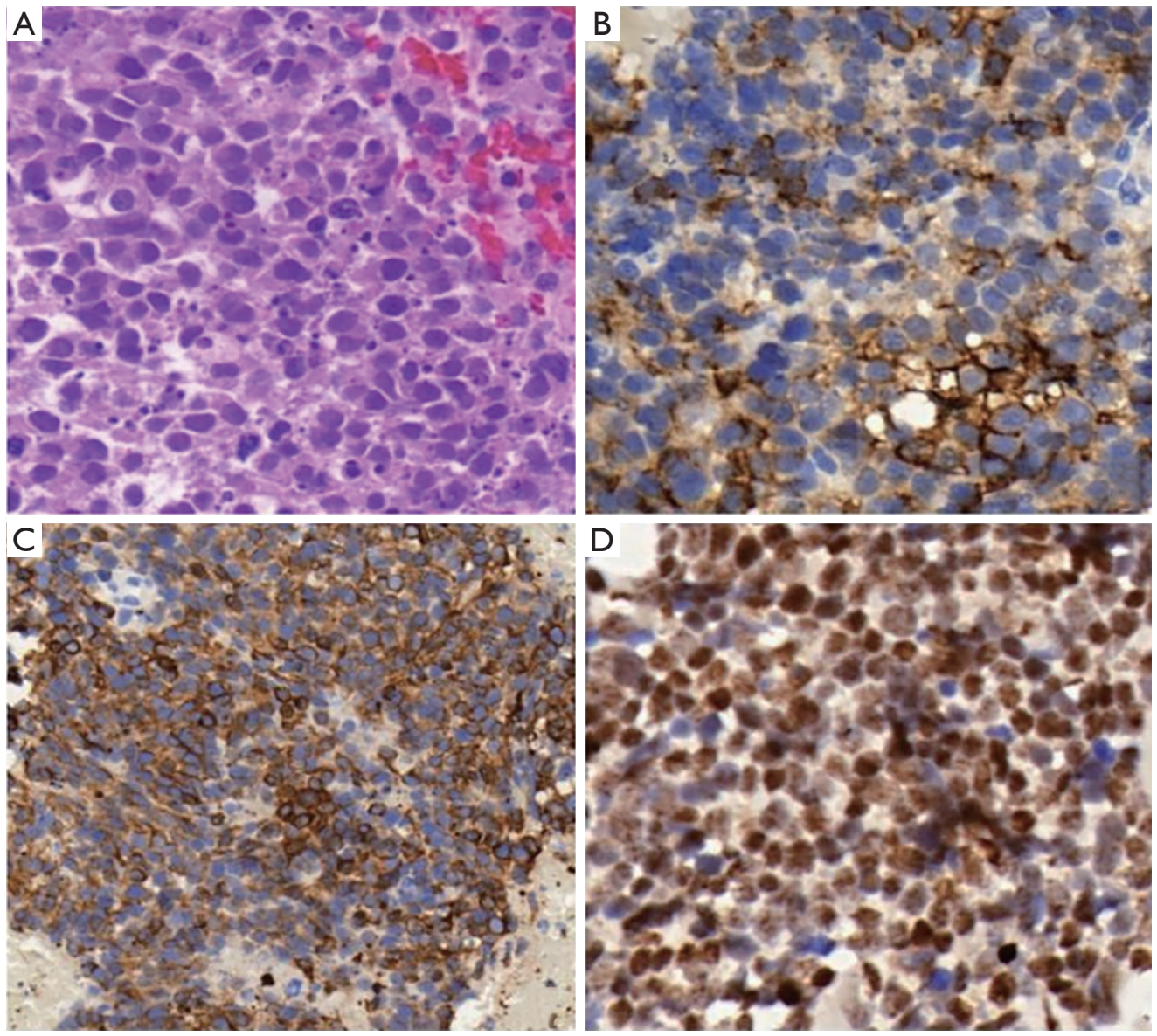

Figure 2 Expression of CD56, CKpan and Nova1 in SCLC. (A) SCLC (HE, ×400), (B) expression of CD56 in SCLC (IHC, ×400), (C) expression of CKpan in SCLC (IHC, ×200), (D) expression of Nova1 in SCLC (IHC, ×400). SCLC, small-cell lung cancer.

Table 1 Comparison of Nova1 expression between small-cell lung cancer (SCLC) and non-small cell lung cancer (NSCLC) or their lymph node/ liver metastases

\begin{tabular}{llcccc}
\hline \multirow{2}{*}{ Group } & Tissue & \multicolumn{3}{c}{ Nova1 expression } \\
\cline { 3 - 5 } & & Samples & Negative & Low & High \\
\hline \multirow{2}{*}{ SCLC } & Primary lesion & 84 & 7 & 61 \\
& Lymph node/liver metastasis & 16 & 5 & 4 & 7 \\
\multirow{2}{*}{ NSCLC } & Primary lesion & 25 & 25 & 0 & 0 \\
& Lymph node/liver metastasis & 5 & 5 & 0 \\
\hline
\end{tabular}

(7-10). Zhang et al. $(11,12)$ showed that expression of Nova1 in hepatocellular carcinoma Huh7 and SMMC7721 cell lines was significantly higher than that in normal hepatocyte cell lines. Moreover, down-regulation of Nova1 expression resulted in decreased proliferation and invasion of Huh7 cells, whereas Nova1 overexpression enhanced proliferation and invasion in SMMC-7721 cells. Similar results were also obtained in a nude mouse model of primary liver cancer. They further compared Nova1 expression in cancerous tissue and adjacent normal tissue of 91 patients with hepatocellular carcinoma and found that Nova1 expression was higher in the cancerous tissue, 

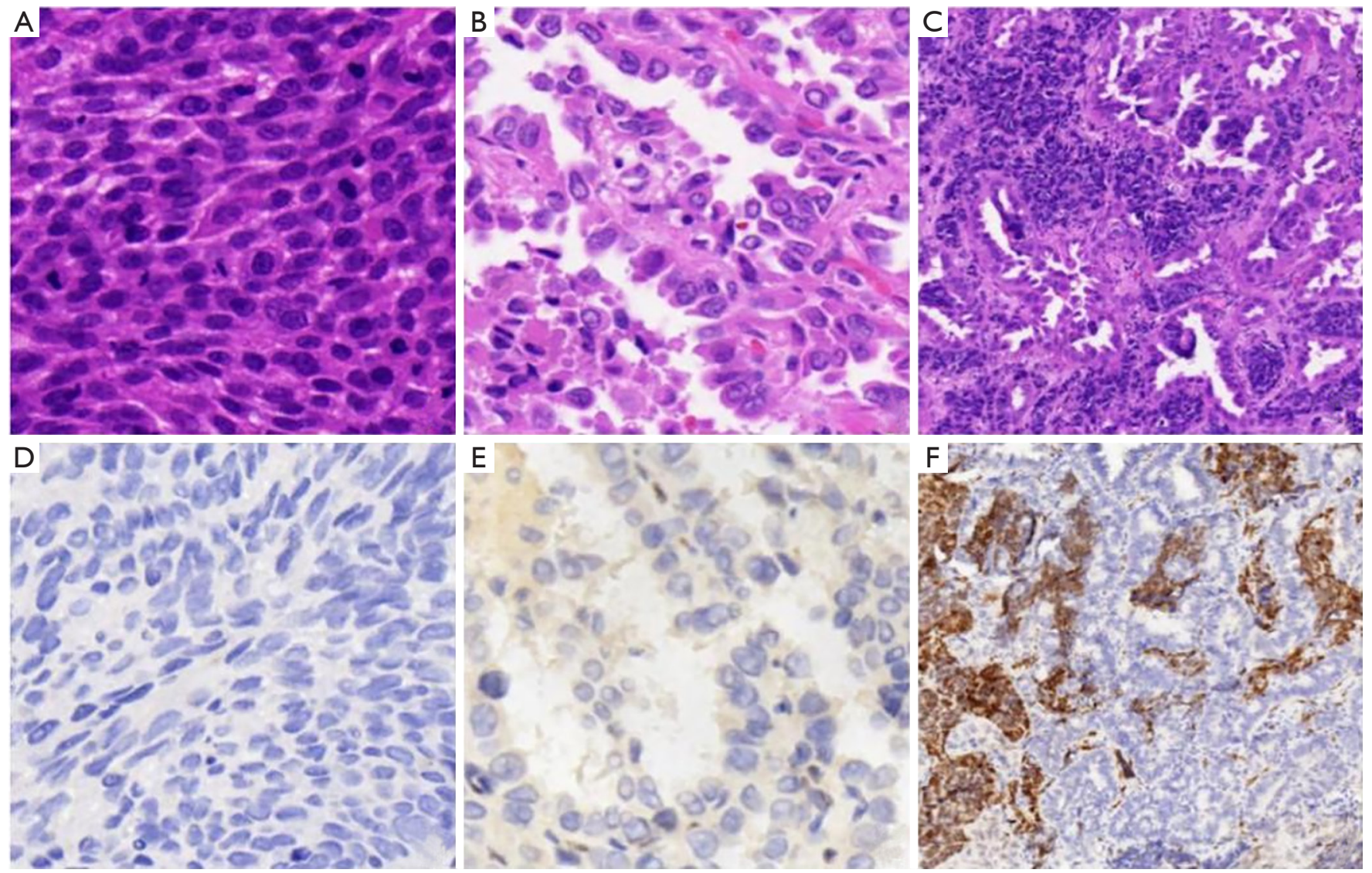

Figure 3 Expression of Nova1 in NSCLC. (A) Squamous cell carcinoma (HE, $\times 400)$, (B) adenocarcinoma (HE, $\times 400)$, (C) SCLC complicated with adenocarcinoma (HE, $\times 100)$, (D) nova1 was negative in squamous cell carcinoma (IHC, $\times 400),(\mathrm{E})$ nova1 expression was negative in adenocarcinoma (IHC, $\times 400)$, (F) Nova1 expression was strongly positive in small-cell lung carcinoma cells, which showed a pattern of nucleocytoplasmic staining, but expression was negative in adenocarcinoma (IHC, $\times 100)$. SCLC, small-cell lung cancer.

Table 2 Comparison of Nova1, CD56, SYN, CgA, P63 and P40 expression in 100 samples of small-cell carcinoma

\begin{tabular}{lccc}
\hline Marker & Negative & Low & High \\
\hline Nova1 & 12 & 20 & 68 \\
CD56 & 8 & 29 & 63 \\
SYN & 25 & 31 & 44 \\
CgA & 21 & 24 & 55 \\
P63 & 87 & 3 & 10 \\
P40 & 93 & 2 & 5 \\
\hline
\end{tabular}

and higher Nova1 expression was correlated with poorer prognosis, representing an independent risk factor for patient survival and tumour recurrence. Yu et al. (13) used immunohistochemistry to examine three cases of malignant melanoma and two cases of intradermal naevus and found that all the malignant melanoma specimens expressed Nova1, while all the intradermal naevus specimens were Nova1-negative. Suppression of Nova1 expression in melanoma cell lines inhibited proliferation, metastasis and invasion in melanoma A375 and A385 cells, while overexpressing Nova1 had the opposite effect. Zhi et al. (14) demonstrated that Nova1 is regulated by miR-181b$5 \mathrm{p}$ in astrocytoma, and elevated Nova 1 expression in this tumour promotes cell proliferation and invasion and inhibits apoptosis; when miR-181b-5p was overexpressed in astrocytoma, Noval expression was significantly downregulated, cell proliferation and invasion were inhibited and the apoptosis rate was significantly increased. Yet, Kim et al. (8) demonstrated that Nova1 was significantly 
downregulated in gastric cancer and gastric cancer cell lines, and lower Nova1 expression was closely related to poorer prognosis in patients with gastric cancer. So far, there has been no report about Nova1 expression in SCLC. In this study, it was found that Novalexpression was significantly higher in the SCLC cell line H446 than in the bronchial epithelial cell line BEAS-2B and lung adenocarcinoma cell lines A549 and H1299.Immunohistochemistry analysis also demonstrated that Nova 1 was expressed in up to $88 \%$ of SCLC specimens.

\section{The role of Nova1 in pathological diagnosis of SCLC}

Traditional neuroendocrine markers include CD56, SYN, $\mathrm{CgA}$, and the like; among them, CD56 is mainly expressed in relatively undifferentiated neuroendocrine tumours, which is helpful for the diagnosis of SCLC. Kontogianni et al. (15) and Yeh and Chou (16) reported that $97-100 \%$ of SCLCs express CD56, and in this group, the rate of CD56 positivity was $92 \%$. However, CD56 is also expressed in NK cells, SCC, adenocarcinoma and mesothelioma, showing poor specificity. Syn has relatively low sensitivity for SCLCs, and CgA is often not expressed in relatively undifferentiated SCLCs, which contain fewer cytoplasmic secretory granules $(16,17)$. In recent years, scholars have found that the commonly used squamous cell marker p63 is present in $30-51 \%$ of lung adenocarcinomas (18) and in $0-76 \%$ of SCLCs $(18-20)$. In this study, the rate of $\mathrm{p} 63$ positivity was $13 \%$, similar to the value of $14 \%$ obtained by Hamanaka et al. (21). P40 is highly sensitive and specific for SCC and is thus considered the best marker for this cancer. However, Nakajima's research shows that although p40 was not diffusely expressed in any of the SCLC cases, the rate of $\mathrm{P} 40$ positivity in small-cell carcinoma can be as high as $34.1 \%$ (22). The rate of $\mathrm{P} 40$ positivity in this group of SCLC patients was 7\%, which was higher than that reported by Tatsumori et al. 5\% (18). At present, no studies on Nova1 expression in SCLC have been reported. In this study, the rate of Nova1 positivity in SCLC patients was $88 \%$, as determined by the presence of brown staining in immunohistochemistry, and $72.3 \%$ of cases showed high levels of Nova1 expression. Nova1 was not expressed in non-neuroendocrine tumours such as SCC and adenocarcinoma, suggesting that Nova1 is highly specific for SCLC. In addition, SCLC is a highly malignant tumour with extremely high mortality and poor prognosis, mostly due to its invasive tumour growth and metastasis. A number of in vitro studies have shown that abnormal activation of
Nova1 can promote cell differentiation and proliferation, inhibit cell apoptosis and promote tumour cell metastasis, suggesting that Nova1 expression is negatively correlated with prognosis and can be used as an independent prognostic marker. It should be noted that although Nova1 is also highly expressed in hepatocellular carcinoma, malignant melanoma, astrocytoma and oligodendroglioma, they can be differentiated from SCLC in terms of the primary tumour site, histomorphology and other immune markers, so it does not undermine the clinical value of Nova1 as a specific marker for SCLC.

In summary, RT-PCR showed that mRNA levels of Nova1 were significantly increased in SCLC cells relative to normal bronchial epithelial cells, adenocarcinoma cells and SCC cells. Further immunohistochemical examination of paraffin- embedded specimens 100 SCLCs confirmed that Nova1 can be used as a novel neuroendocrine marker for clinical pathological diagnosis of SCLC. Moreover, compared with traditional neuroendocrine markers, Nova1 has high sensitivity and specificity, in addition to its role as a predictor of recurrence and prognosis.

\section{Acknowledgments}

Funding: None.

\section{Footnote}

Conflicts of Interest: All authors have completed the ICMJE uniform disclosure form (available at http://dx.doi. org/10.21037/tcr.2019.12.99). The authors have no conflicts of interest to declare.

Ethical Statement: The authors are accountable for all aspects of the work in ensuring that questions related to the accuracy or integrity of any part of the work are appropriately investigated and resolved. The study was conducted in accordance with the Declaration of Helsinki (as revised in 2013). This research was approved by the Medical Ethics Committee of Shanghai East Hospital, Tongji University School of Medicine. All specimens were obtained with informed consent from the patient.

Open Access Statement: This is an Open Access article distributed in accordance with the Creative Commons Attribution-NonCommercial-NoDerivs 4.0 International License (CC BY-NC-ND 4.0), which permits the noncommercial replication and distribution of the article with 
the strict proviso that no changes or edits are made and the original work is properly cited (including links to both the formal publication through the relevant DOI and the license). See: https://creativecommons.org/licenses/by-nc-nd/4.0/.

\section{References}

1. Bamard WG. The nature of the "oat-celled sarcoma" of the mediastinum. J Pathol Bacteriol 1926;29:241-4.

2. Schmittel A. Controversies in the treatment of advanced stages of small cell lung cancer. Front Radiat Ther Oncol 2010;42:193-7.

3. Ferlay J, Steliarova-Foucher E, Lortet-Tieulent J, et al. Cancer incidence and mortality patterns in Europe: estimates for 40 countries in 2012. Eur J Cancer 2013;49:1374-403.

4. Travis WD. Update on small cell carcinoma and its differentiation from squamous cell carcinoma and other non-small cell carcinomas. Mod Pathol 2012;25 Suppl 1:S18-30.

5. Buckanovich RJ, Posner JB, Darnell RB. Nova, the paraneoplastic Ri antigen, is homologous to an RNAbinding protein and is specifically expressed in the developing motor system. Neuron 1993;11:657-72.

6. Darnell RB, Posner JB. Paraneoplastic syndromes involving the nervous system. N Engl J Med 2003;349:1543-54.

7. Kim EK, Yoon SO, Kim SH, et al. Upregulated neurooncological ventral antigen 1 (NOVA1) expression is specific to mature and immature T- and NK-cell lymphomas. J Pathol Transl Med 2016;50:104-12.

8. Kim EK, Yoon SO, Jung WY, et al. Implications of NOVA1 suppression within the microenvironment of gastric cancer: association with immune cell dysregulation. Gastric Cancer 2017;20:438-47.

9. Liu N, Yang J, Yuan R, et al. Effects of miR-181a on the biological function of multiple myeloma. Oncol Rep 2019;42:291-300.

10. Jin L, Li H, Wang J, et al. MicroRNA-193a-5p exerts a tumour suppressor role in glioblastoma via modulating NOVA1. J Cell Biochem 2019;120:6188-97.

11. Zhang YA, Liu HN, Zhu JM, et al. RNA binding protein Nova1 promotes tumor growth in vivo and its potential mechanism as an oncogene may due to its interaction with GABAA Receptor- $\gamma 2$. J Biomed Sci 2016;23:71.

12. Zhang YA, Zhu JM, Yin J, et al. High expression of neurooncological ventral antigen 1correlates with poor prognosis in hepatocellular carcinoma. PLoS One 2014;9:e90955.

13. Yu X, Zheng H, Chan MTV, et al. NOVA1 acts as an oncogene in melanoma via regulating FOXO3a expression. J Cell Mol Med 2018;22:2622-30.

14. Zhi F, Wang Q, Deng D, et al. MiR-181b-5p downregulates NOVA1 to suppress proliferation, migration and invasion and promote apoptosis in astrocytoma. PLoS One 2014;9:e109124.

15. Kontogianni K, Nicholson AG, Butcher D, et al. CD56: a useful tool for the diagnosis of small cell lung carcinomas on biopsies with extensive crush artefact. J Clin Pathol 2005;58:978-80.

16. Yeh YC, Chou TY. Pulmonary neuroendocrine tumours: study of 90 cases focusing on clinicopathological characteristics, immunophenotype, preoperative biopsy, and frozen section diagnoses. J Surg Oncol 2014;109:280-6.

17. Yun JP, Zhang MF, Hou JH, et al. Primary small cell carcinoma of the esophagus: clinicopathological and immunohistochemical features of 21 cases. BMC Cancer 2007;7:38.

18. Tatsumori T, Tsuta $\mathrm{K}$, Masai $\mathrm{K}$, et al. $\mathrm{p} 40$ is the best marker for diagnosing pulmonary squamous cell carcinoma: comparison with p63, cytokeratin 5/6, desmocollin-3, and sox2. Appl Immunohistochem Mol Morphol 2014;22:377-82.

19. Au NHC, Gown AM, Cheang M, et al. P63 expression in lung carcinoma: a tissue microarray study of 408 cases. Appl Immunohistochem Mol Morphol 2004;12:240-7.

20. Zhang C, Schmidt LA, Hatanaka K, et al. Evaluation of napsin A, TTF-1, p63, p40, and CK5/6

immunohistochemical stains in pulmonary neuroendocrine tumors. Am J Clin Pathol 2014;142:320-4.

21. Hamanaka W, Motoi N, Ishikawa S, et al. A subset of small cell lung cancer with low neuroendocrine expression and good prognosis: a comparison study of surgical and inoperable cases with biopsy. Hum Pathol 2014;45:1045-56.

22. Nakajima N, Yoshizawa A, Moriyoshi K, et al. P40 expression in small cell lung cancer: The presence of p40-positive cells does not always indicate squamous differentiation. Thorac Cancer 2019;10:1188-92.

Cite this article as: Deng S, Liu M, Xiao T, Gao J. Expression of neuro-oncological ventral antigen-1 in small-cell lung cancer and its value in pathological diagnosis. Transl Cancer Res 2020;9(2):1144-1150. doi: 10.21037/tcr.2019.12.99 